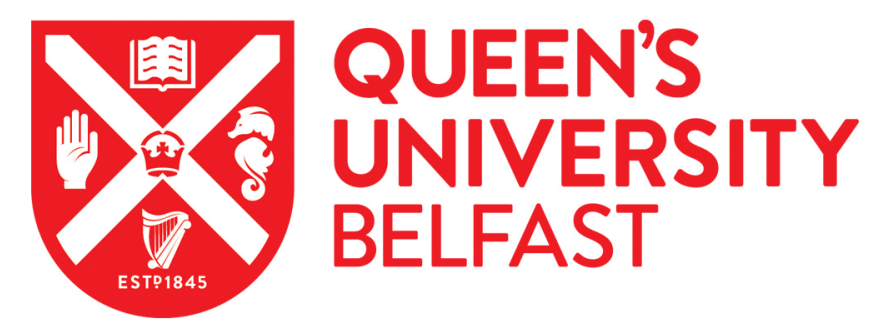

\title{
Off-colony screening of biosynthetic libraries by rapid laser-enabled mass spectrometry
}

Gowers, G-O. F., Cameron, S. J. S., Perdones-Montero, A., Bell, D., Chee, S. M., Kern, M., Tew, D., Ellis, T., \& Takats, Z. (2019). Off-colony screening of biosynthetic libraries by rapid laser-enabled mass spectrometry. ACS Synthetic Biology. https://doi.org/10.1021/acssynbio.9b00243

Published in:

ACS Synthetic Biology

Document Version:

Peer reviewed version

Queen's University Belfast - Research Portal:

Link to publication record in Queen's University Belfast Research Portal

Publisher rights

Copyright $\odot 2019$ American Chemical Society. This work is made available online in accordance with the publisher's policies. Please refer to any applicable terms of use of the publisher.

\section{General rights}

Copyright for the publications made accessible via the Queen's University Belfast Research Portal is retained by the author(s) and / or other copyright owners and it is a condition of accessing these publications that users recognise and abide by the legal requirements associated with these rights.

Take down policy

The Research Portal is Queen's institutional repository that provides access to Queen's research output. Every effort has been made to ensure that content in the Research Portal does not infringe any person's rights, or applicable UK laws. If you discover content in the Research Portal that you believe breaches copyright or violates any law, please contact openaccess@qub.ac.uk. 
Off-colony screening of biosynthetic libraries by rapid laser-enabled mass spectrometry

Glen-Oliver F. Gowers ${ }^{1,2, \dagger}$, Simon J.S. Cameron ${ }^{3,6, \dagger}$, Alvaro Perdones-Montero ${ }^{3,6}$, David Bell $^{4}$, Soo Mei Chee ${ }^{4}$, Marcelo Kern ${ }^{5}$, David Tew ${ }^{5}$, Tom Ellis ${ }^{1,2, *}$, Zoltan Takáts ${ }^{3,6, *}$

${ }^{1}$ Imperial College Centre for Synthetic Biology (IC-CSynB), Imperial College London, London, SW7 2AZ, UK

${ }^{2}$ Department of Bioengineering, Imperial College London, London, SW7 2AZ, UK

${ }^{3}$ Section of Computational and Systems Medicine, Department of Surgery and Cancer, Imperial College London, London, SW7 2AZ, UK

${ }^{4}$ SynbiCITE, Imperial College London, London, SW7 2AZ, UK

${ }^{5}$ GlaxoSmithKline, Stevenage, SG1 2NY, UK

${ }^{6}$ Ambimass, London, W12 0BZ

†These authors contributed equally to this work. ${ }^{*}$ Correspondence should be addressed to Z.T. (z.takats@imperial.ac.uk) or T.E. (t.ellis@imperial.ac.uk)

Keywords: high throughput screening, ambient mass spectrometry, synthetic biology, metabolic engineering, REIMS 


\section{Abstract}

Leveraging advances in DNA synthesis and molecular cloning techniques, synthetic biology increasingly makes use of large construct libraries to explore large design spaces. For biosynthetic pathway engineering the ability to screen these libraries for a variety of metabolites of interest is essential. If the metabolite of interest or the metabolic phenotype is not easily measurable, screening soon becomes a major bottleneck involving timeconsuming culturing, sample preparation, and extraction. To address this, we demonstrate the use of automated Laser-Assisted Rapid Evaporative Ionisation Mass Spectrometry (LAREIMS) - a form of ambient laser desorption ionisation mass spectrometry - to perform rapid mass spectrometry analysis direct from agar plate yeast colonies without sample preparation or extraction. We use LA-REIMS to assess production levels of violacein and betulinic acid directly from yeast colonies at a rate of 6 colonies per minute. We then demonstrate the throughput enabled by LA-REIMS by screening over 450 yeast colonies in under 4 hours, while simultaneously generating recoverable glycerol stocks of each colony in real-time. This showcases LA-REIMS as a pre-screening tool to complement downstream quantification methods such as LCMS. Through pre-screening several hundred colonies with LA-REIMS, we successfully isolate and verify a strain with a 2.5 -fold improvement in betulinic acid production. Finally, we show that LA-REIMS can detect 20 out of a panel of 27 diverse biological molecules, demonstrating the broad applicability of LA-REIMS to metabolite detection. The rapid and automated nature of LA-REIMS makes this a valuable new technology to complement existing screening technologies currently employed in academic and industrial workflows. 
Screening biomass samples for a metabolite-of-interest is a critical component in many areas of bioscience and biotech research, however, its importance is particularly strong in the field of synthetic biology. Here, large libraries of DNA-encoded biosynthesis pathways are introduced to industrially-suited host organisms where a large number of features can be genetically altered with the aim of maximising production of a particular metabolite. The commercial production of natural products and other metabolites using engineered microorganisms has shown remarkable success. Industrial model organisms, such as Saccharomyces cerevisiae, have been used to produce therapeutically-important molecules such as artemisinic acid ${ }^{1}$, morphine precursors (thebaine and hydrocodone) ${ }^{2}$, and benzylpenicillin ${ }^{3}$.

With a plethora of new tools from synthetic biology, it is now becoming trivial to introduce and express heterologous genes in industrial host organisms. However, there remains the major problem of achieving economically viable titre, rate, and yield ${ }^{4-6}$. Improvements in the accessibility, cost, and scale of DNA synthesis and assembly means that it is increasingly common to build sizeable DNA libraries to explore large design spaces for a biosynthesis pathway of interest ${ }^{7}$. In addition, strain optimisation can employ rapid modification tools such as CRISPR $/$ cas $^{8}$ and genome diversification methods such as random mutagenesis ${ }^{9}$ or SCRaMbLE (Synthetic Chromosome Rearrangement and Modification by LoxP-mediated Evolution $)^{10-18}$ which further increase the design space that can be explored. Screening for the highest producers from the large libraries that result from these approaches becomes a major bottleneck, particularly when a metabolite does not confer an easily detectable phenotype such as colour or growth effect ${ }^{19}$.

The current standard for metabolite detection is therefore liquid or gas chromatographymass spectrometry (LCMS or GCMS) ${ }^{19}$. However, these methods typically require time consuming steps of sample preparation, liquid growth, liquid or solid phase extraction methods (using tailored solvents for different metabolites), and optimisation of the column 
and the MS parameters as well. The time demand associated with these steps scales with the number of strains to be screened, rapidly becoming a major bottleneck for pathway and strain engineering. Attempts to address this bottleneck have made use of biosensors ${ }^{19-21}$ which allow for extremely high throughput screening using fluorescence-activated cell sorting (if metabolite output triggers fluorescence) or selection (if output promotes survival). However, the use of biosensors is predicated on significant prior development and characterisation that must be customised for each metabolite ${ }^{21}$. Biosensors are usually only suitable for either extracellular or intracellular metabolites, and the latter can be complicated by subcellular compartmentalisation.

Alternatively, droplet-mass spectrometry can detect secreted metabolites and the use of mass spectrometry means virtually any metabolite can be detected. However, this technology requires custom microfluidics and significant optimisation to be an effective screen $^{22}$. Other mass spectrometry techniques exist that can rapidly analyse solid biomass samples with minimal sample preparation such as (nano) DESI-MS (desorption electrospray ionisation) ${ }^{23-28}$, LESA-MS (liquid extraction surface analysis) ${ }^{29-31}$, MALDI-MS (matrix assisted laser desorption ionisation) $)^{32-34}$, and DI-MS (direct infusion) ${ }^{35-37}$ though such methods require a level of sample preparation or modification that naturally limits throughput (summarised in Supplementary Table 6). There remains a clear need for an automated and high-throughput screening method that can fit into a standard strain engineering workflow, as a first-pass qualitative screen that is sample agnostic and requires no sample preparation or modification, to reduce the number of strains for subsequent quantification.

Rapid Evaporative Ionisation Mass Spectrometry (REIMS) is an established method that has previously been shown to detect metabolic profiles in a wide variety of samples including various human tissues ${ }^{38,39}$, and microorganisms ${ }^{40-43}$. The technology was originally implemented as a direct combination of electrosurgical tissue ablation and mass spectrometric analysis of the resulting aerosol plume. Although the method has strong 
surgical roots, it has also been utilized for the rapid identification of clinical microbiology isolates including pathogenic yeasts ${ }^{40,41,43}$. Unlike commercially available MS platforms, REIMS requires no sample preparation.

Here, we describe an approach that uses modified high-throughput REIMS (LA-REIMS) technology where a high-powered laser evaporates a portion of an S. cerevisiae colony $(0.8$ $\mathrm{mm}^{2}$ ) to enable immediate semi-quantitative assessment of metabolites from engineered strain libraries. Such a small laser burn diameter minimises any variation as a result of colony size disparity (Supplementary Figure 6). This method offers a new tool for high throughput library screening by allowing metabolite measurement directly from individual $S$. cerevisiae colonies with no sample preparation or liquid culturing, providing significantly higher throughput than other mass spectrometry methods. We demonstrate the potential for LA-REIMS to be used as a first-pass screen for cells engineered to produce a therapeutically relevant metabolite with no known screening method beyond commercially available mass spectrometry. Furthermore, we show that LA-REIMS has the potential to screen for a wide variety of other biologically-relevant metabolites.

\section{Results}

\section{Validating LA-REIMS via analysis of violacein-producing strains.}

Laser-assisted-REIMS (LA-REIMS) is a modified version of REIMS using a $\mathrm{CO}_{2}$ laser to generate sample aerosol without necessitating contact with the sample - allowing substantially increased throughput as no cleaning or changing of probe is required between samples ${ }^{44}$, (Fig 1A). The resulting aerosol, containing gas phase ions of metabolites, among other biological molecules such as structural lipids, is introduced into a mass spectrometer for analysis within one second of ablation, offering real-time semi-quantitative data on metabolite levels. This technology requires no sample preparation or liquid culturing. Colonies are optically detected on the agar plate and automatically selected by an 
automated algorithm. The laser beam is delivered by a hollow fibre light guide (OmniGuide) directly mounted on the colony picker arm of the robotic platform (TECAN Freedom Evo, with SciRobotics Pickolo add-on). Following selection, colonies of interest are automatically scanned with real-time data collection allowing analysis of a colony in under ten seconds (Fig 1B).

To first determine if LA-REIMS could identify biomass samples containing high levels of a heterologous metabolite, we chose to test it on an engineered yeast library where the biosynthesised natural product could also be easily screened. Violacein was selected as it is a colorimetrically detectable product. If LA-REIMS is to be successful, we would expect that the highest producers of violacein (i.e. the darkest colonies) would generate the strongest signal peak intensities on the mass spectra, while lower producer colonies give weaker signals.

Violacein biosynthesis involves five heterologous genes (VioA-E, Supplementary Figure $1 \mathrm{~A})^{11}$ which we assembled together using modular DNA cloning into a CEN/ARS plasmid transformed into yeast. Each gene was either expressed from a low- or a high-strength promoter, resulting in a combinatorial library of 32 yeast strains (Supplementary Figure 1B and construction detailed in Supplementary Tables 1-4). This library was streaked onto selective agar media to produce individual colonies. The streaked library was analysed by LA-REIMS ( $n=7$ colonies screened) and the images of culture lawns (saturated culture spotted onto agar) compared to analysis results (Fig 1C).

In the LA-REIMS analysis (Fig 1C) we see a visible enrichment for the darkest colonies at higher LA-REIMS values. However, a perfect correlation for all samples is not seen, which we attribute to the fact that the LA-REIMS signal for violacein is relatively weak and so subject to more influence from noise and from overlap with other naturally-produced metabolites present in the colony (Fig 1D, Supplementary Figure 3). Nonetheless, the top 
$20 \%$ of strains screened by REIMS represent significantly darker cultures than the remaining strains screened (Supplementary Figure 5) and we were therefore confident that LA-REIMS enriches for higher producers. Considering its speed and ease we decided it could provide a useful new tool to complement quantitative chromatography-based mass spectrometry despite a relatively high false positive and negative discovery rate. In particular, it offers a rapid and easy initial screening method to identify a subset of strains from a library that are more likely to have higher levels of the metabolite-of-interest, or several metabolites, and so can be taken forward for more quantitative analysis.
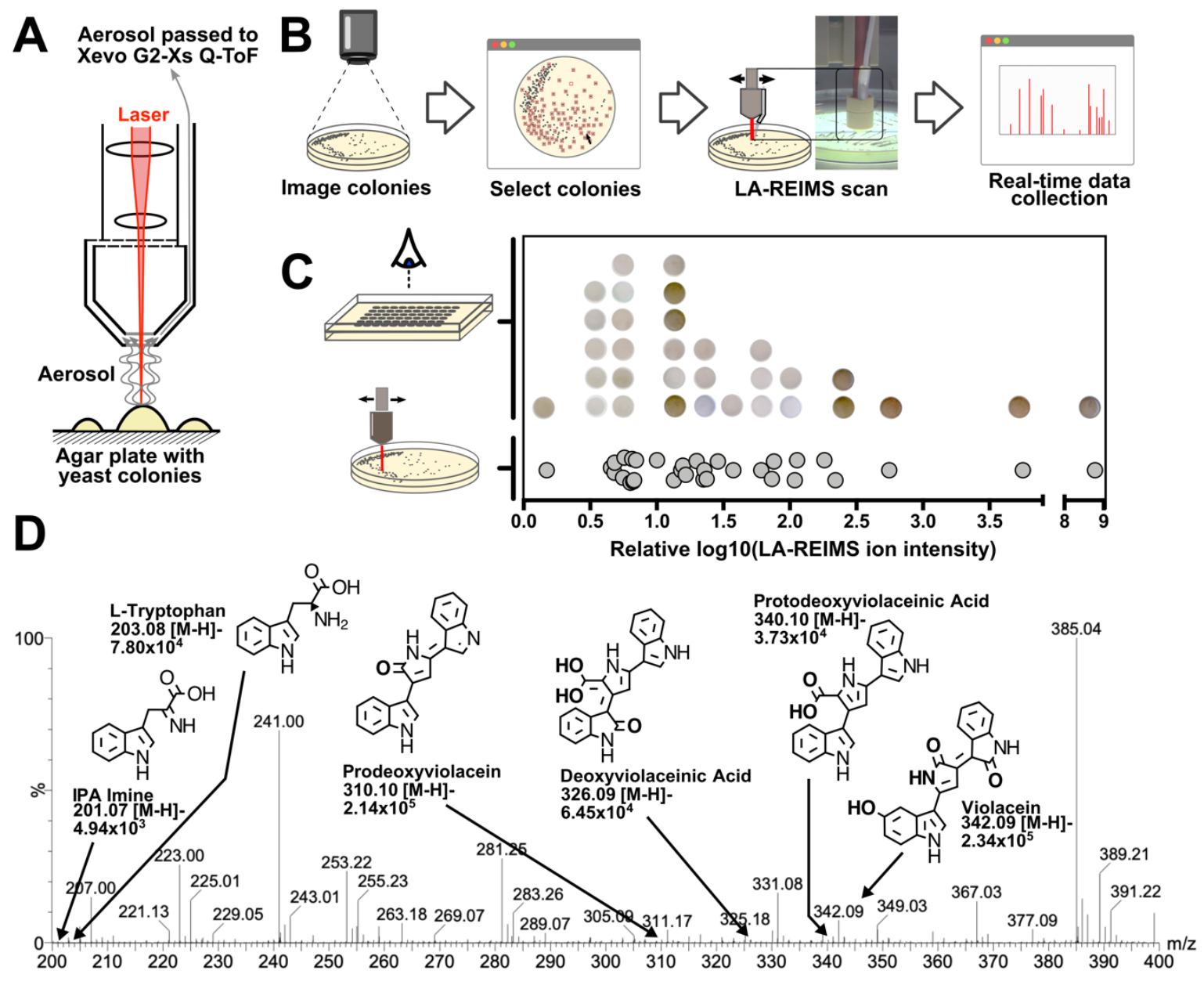

Fig. 1 Investigating the validity of REIMS for the semi-quantitative detection of violacein. $\mathbf{A}$. Diagram illustrating the principle of LA-REIMS. A $\mathrm{CO}_{2}$ laser (red) rapidly heats a portion of biomass (yellow) and the resulting aerosol is aspirated into a Xevo G2-XS QToF mass spectrometer by the instrument's native vacuum. B. A schematic illustrating the automated workflow for LA-REIMS. As presented here this takes as little as 10 seconds per colony. $\mathbf{C}$. A violacein-producing strain library was constructed (32 strains, Supplementary Figure 1B and Supplementary Tables 1-4). Each strain was grown to saturation and $10 \mu$ spotted onto 
agar. After 2 days of growth the culture spots were photographed on a light box (white/black circles, above). Culture lawn darkness was used as a proxy for violacein concentration ${ }^{11}$. Separately the same strains were streaked out on agar and grown for 2 days to form colonies. Mean REIMS ion integration values $(n=7)$ are shown relative to a negative control (strain without the violacein pathway). D. Example of a portion of the full spectra (200-400 $\mathrm{m} / \mathrm{z}$ ) collected in real-time during LA-REIMS analysis of yGG074. Locations of peaks corresponding to intermediate metabolites in the biosynthetic pathway are indicated (Supplementary Figure 1A) to illustrate that LA-REIMS can analyse multiple metabolite peaks simultaneously. Each metabolite is named, the $[\mathrm{M}-\mathrm{H}]-$ value shown, and the peak intensity shown.

\section{Betulinic acid detection by LA-REIMS}

We intended to demonstrate the use of LA-REIMS as a screen for a heterologous metabolite that is not coloured, fluorescent, nor affects growth. Betulinic acid (BA) is an industrially relevant triterpenoid with therapeutic potential ${ }^{45-47}$. With no sustainable and economically scalable source of BA, biosynthesis by microbial organisms amenable to bioprocessing is of great interest. Crucially for this study there is no published screening method for BA beyond chromatographic/mass spectrometric methods.

First, we established a standard curve for BA measurement from solid yeast colonies. For LA-REIMS to work as a qualitative first-pass screen we expect a linear correlation between standard BA concentrations and the resulting signal peak intensities. Saturated liquid $S$. cerevisiae cultures $(10 \mu \mathrm{l})$ were spotted onto agar and grown for 2 days $\left(30^{\circ} \mathrm{C}\right) .5 \mu \mathrm{lBA}$ standard (dissolved in DMSO) was then physically added onto each spotted culture and allowed to dry for 5 minutes prior to LA-REIMS analysis $(n=3)$. Spotting the standard onto a culture aimed to replicate the natural extracellular accumulation of betulinic acid (Supplementary Figure 7). Negligible signal was observed at the BA [M-H]- peak $(\mathrm{m} / \mathrm{z}$ 455.35) for the negative control (yeast with DMSO, Supplementary Figure 4). For the rest, a linear correlation was observed over the entire range of BA standards used $\left(2-2 \times 10^{-5} \mathrm{~g} / \mathrm{L}\right)$ with an $R^{2}=0.967$ (Fig 2A). This gave us confidence that LA-REIMS could be used to screen for biosynthesised BA in yeast strains and identify the highest producers for further quantitative analysis. 
To next verify that LA-REIMS could detect BA that has been biosynthesised by yeast we needed to compare LA-REIMS and LCMS peak intensities from the same colony biomass samples and see a linear relationship between both analysis methods. To do this, we constructed a panel of 36 yeast strains engineered for the biosynthesis of BA using modular assembly to make combinations of the two genes expressing enzymes essential for BA synthesis (BPLO and AtLUS1) ${ }^{48}$ and two genes (tHMG1 and ERG9) expressing enzymes known to improve flux through the mevalonate pathway ${ }^{49-52}$. As before, both strong and weak promoters were used to make the combinatorial plasmid library. This plasmid library was transformed into a haploid yeast strain containing an additional P450 reductase enzyme, AtATR1 ${ }^{48}$ (Supplementary Figure 2 and construction described in Supplementary Tables 1-4).

The 36 strains were grown to saturation in liquid culture $\left(\mathrm{SDO} U \mathrm{UA}^{-}\right)$, spotted onto selective media agar and grown for 2 days $\left(30^{\circ} \mathrm{C}\right)$. After first screening by LA-REIMS, the same spotted cultures were lifted from the agar using a loop and resuspended in $500 \mu l$ water and pelleted, prior to subsequent BA extraction using two propan-2-ol wash steps, then LCMS analysis. This sample prep approach was taken to avoid any biological differences that might be introduced through differing growth conditions between colony growth for LAREIMS vs standard liquid culture growth for LCMS, such as nutrient supply and aeration.

LCMS and LA-REIMS measurements for each strain were then directly compared (Fig 2B, $\mathrm{n}=3$ biological repeats). Promisingly, a statistically significant correlation between LCMS and LA-REIMS analysis is seen (Fig $\left.2 B, R^{2}=0.613, P<0.0001\right)$. This indicates that LA-REIMS is capable of acting as a first-pass screen with the aim of enriching a set of strains for high producers, in this case yeast strains engineered to biosynthesise BA. 

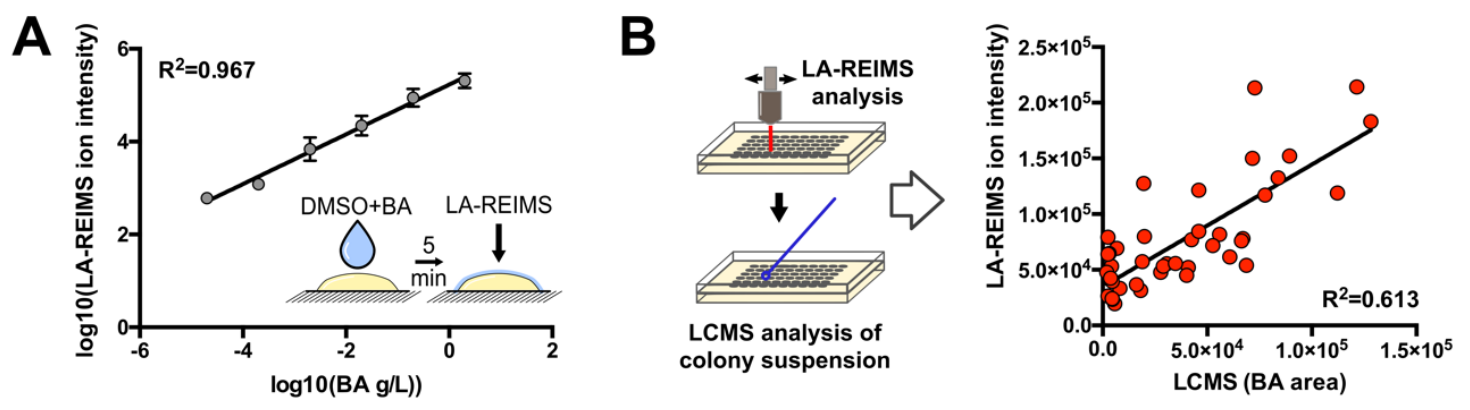

Fig. 2. Investigating the semi-quantitative detection of betulinic acid (BA) by REIMS. A. Wildtype BY4741 was grown to saturation and $10 \mu \mathrm{l}$ spotted onto agar and grown for 2 days to form uniform culture spots. Betulinic acid standard solutions $\left(2-2 \times 10^{-5} \mathrm{~g} / \mathrm{L}\right)$ in DMSO were spotted on and allowed to dry for 5 minutes ( $n=3$, illustrated inset). Error bars indicate standard deviation. Pearson correlation coefficient, two tailed, $r=0.9950(P<0.0001)$ and $R^{2}=0.967$ by linear regression analysis. $B$. Spotted cultures of a betulinic acid library (36 strains, $n=3$, Supplementary Figure 2 and Supplementary Tables 1-4) were screened by REIMS. Immediately after, the same biomass was entirely picked and resuspended in water. Resulting LCMS peak area ( $n=3$ mean) following BA extraction is compared to the REIMS peak intensity ( $n=3$ mean). Pearson correlation coefficient, two tailed, $r=0.783$ and $R^{2}=0.613$ $(\mathrm{P}<0.0001)$.

\section{Pre-screening a strain library demonstrates throughput of LA-REIMS}

Chromatographic-mass spectrometric methods represent the gold standard regarding metabolite quantification, however they are limited in throughput due to the time required for cell culturing, sample preparation, and chromatographic separation. LA-REIMS avoids all sample preparation and chromatographic separation by instead analysing single colonies directly from agar. This significantly improves the throughput of an initial screen, theoretically enabling a researcher to go from colony to data for a plate in around an hour, as opposed to the typical workflow of one to two days (Fig 3A).

To demonstrate this, we made and screened a large library of genetically diverse yeast producing BA. This was achieved by inducing chromosomal rearrangements of a synthetic version of yeast chromosome $\mathrm{V}$ (strain synV) ${ }^{53}$. This system, SCRaMbLE (Synthetic Chromosome Rearrangement and Modification by LoxP-mediated Evolution), was made as part of the Sc2.0 synthetic yeast genome project. SCRaMbLE is a modular gene 
rearrangement and deletion system inherent to synthetic yeast chromosomes built by the Sc2.0 consortium ${ }^{10,54,55}$. Upon induction of Cre recombinase in Sc2.0 strains, recombination events randomly take place between hundreds of loxP sites placed between genes, diversifying the host strain background. In a previous study we showed that SCRaMbLE of strain synV could benefit cells programmed with genetic constructs to either catabolise xylose or biosynthesise violacein. However, this work required screening many colonies, with only approximately $1 \%$ of post-SCRaMbLE colonies showing improved performance ${ }^{11}$.

LA-REIMS therefore offers an ideal tool for post-SCRaMbLE screening, where several hundred colonies can quickly be assessed to identify the top-performing strains that can then be further inspected by the more time-consuming standard liquid-growth LCMS approach. Notably, all SCRaMbLE studies so far have focused on easy-to-screen phenotypes such as production of carotene $e^{14,18}$ and violacein ${ }^{11,18}$, tolerance to caffeine ${ }^{17}$, ethanol, heat, and acetic acid ${ }^{12}$, and utilisation of $x y l o s e$ as a carbon source ${ }^{11}$, so production of BA, with no easy assay, offers a new challenge.

As every post-SCRaMbLE colony is likely to represent a unique strain that may need to be recovered, we adapted the LA-REIMS protocol to now pick a small amount of each colony into selective growth media in 96-well format prior to LA-REIMS analysis. This was enabled by using a second arm on the LA-REIMS liquid handling robot that is capable of picking colonies using tips. Post-analysis, the 96 -well plates were grown overnight and then $50 \%$ glycerol was added to each well (in a $1: 1$ volume) and the plates stocked at $-80^{\circ} \mathrm{C}$. This ensures that every result from LA-REIMS is linked to a well ID in storage plate so that any strains identified as high producers could be recovered at a later stage (Fig 3B).

A yeast strain, yGG066 from the above described BA-producing library containing a synV chromosome ${ }^{53}$, capable of producing BA to a moderate level $\left(0.56 \mu \mathrm{g} \mathrm{ml}^{-1} \mathrm{OD}_{600^{-1}}, 500 \mu \mathrm{l}\right.$ selective media, shaking) (Supplementary Figure 2 and 8, Supplementary Table 4) was 
transformed with a plasmid expressing a Cre recombinase, pSCW11-CreEBD ${ }^{10}$. SCRaMbLE was induced in a growing culture of yGG066-pSCW11-CreEBD. Induction was done for 4 hours with $1 \mu \mathrm{M}$ beta-estradiol before cells were washed and plated on URA- selective agar to maintain the BA biosynthesis plasmid. After 3 days of growth at $30^{\circ} \mathrm{C}$ yeast colonies were screened using LA-REIMS directly from the original agar plate. A total of 66 unSCRaMbLEd control colonies ('unSCR') and 405 SCRaMbLEd colonies ('SCR') were automatically selected, screened and stocked by LA-REIMS at a rate of $\sim 2$ colonies per minute. LAREIMS analysis revealed visibly more variation in BA production in the post-SCRaMbLE samples compared to the controls (Fig 3C, top).

To verify that LA-REIMS pre-screening can enrich a sample for high BA producers, we took the top 5\% (20 strains) and bottom 5\% (19 strains) identified by LA-REIMS and subjected these to traditional LCMS analysis of BA production in liquid growth. While we suspected that BA production would naturally vary between colony solid-growth conditions and shaking liquid-growth conditions, we still expected that a subset of the high-producer colonies, identified by LA-REIMS, would also be high producers in liquid culture when analysed by LCMS. Our results revealed that in the top $5 \%$ selected by LA-REIMS there are, indeed, four strains with significantly increased BA production under liquid growth conditions (unpaired two-tailed t test, $\mathrm{p}<0.05$ ), with one strain showing a $\sim 2.5$-fold improvement in BA production $\left(1.42 \mu \mathrm{g} \mathrm{ml}^{-1} \mathrm{OD}_{600}{ }^{-1}\right.$ compared to $\left.0.56 \mu \mathrm{g} \mathrm{ml}^{-1} \mathrm{OD}_{600^{-1}}\right)$ (Fig 3C). No high producers were found in the bottom $5 \%$.

In our previous study with directly-assayable phenoypes (violacein production, growth on xylose) only $\sim 1 \%$ of randomly selected post-SCRaMbLE strains yielded hits ${ }^{11}$, and recently we have observed a hit rate of only $\sim 5 \%$ when no pre-screen is performed when using LCMS to detect improved BA production. Therefore, without using LA-REIMS as a prescreening tool we would typically expect a set of 20 colonies to at best only identify 1 strain with improved production. Our LA-REIMS selected sample showed 4 high-producers, and so 
gave at least a four-fold enrichment of hits compared to what would normally be expected.

Admittedly, this represents a high false positive discovery rate and likely also a high false negative rate too, as some strains with improved BA production may have evaded LA-

REIMS detection. However, in screening workflows where hit rates are expected to be low (e.g. $1 \%$ ), some inaccuracy in screening is expected to be an acceptable trade-off for speed and cost. As demonstrated here, LA-REIMS provides a quick initial screening tool that enriches hits severalfold in large libraries that require mass spectrometry analysis.

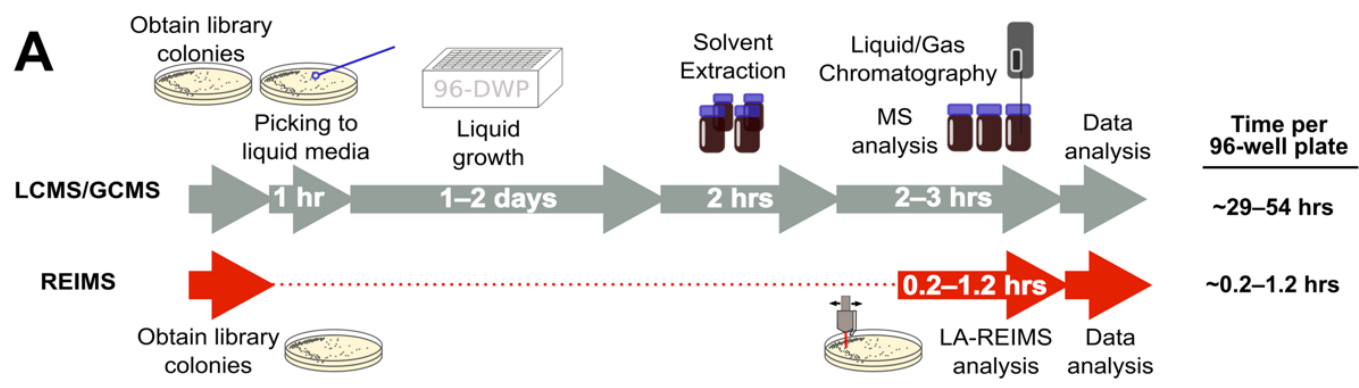

B
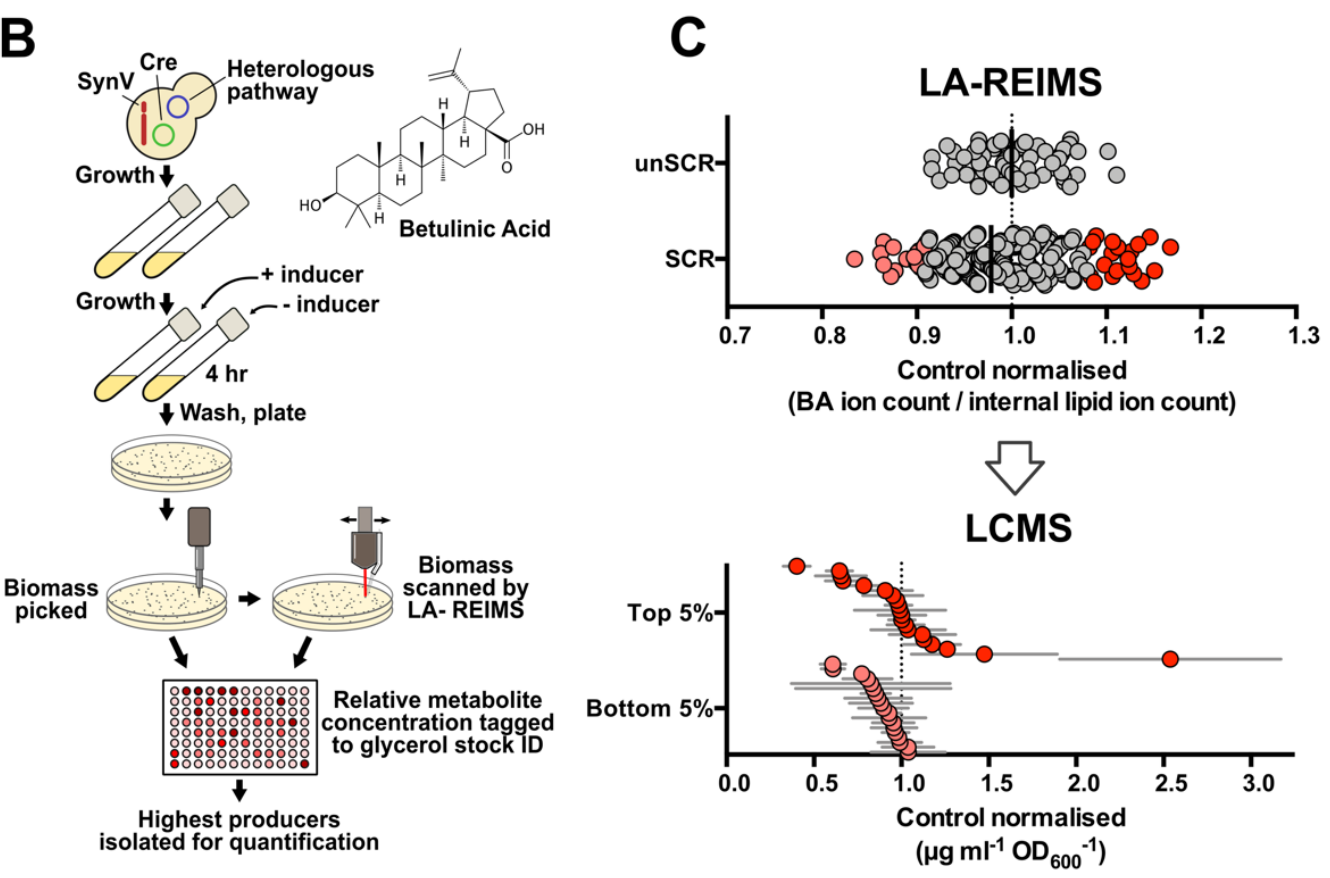

Fig. 3 Demonstrating the potential of REIMS as a first-pass screen for a large strain library. A. A standard commercial chromatography mass spectrometry workflow (LCMS/GCMS) is compared to a standard REIMS workflow, as performed in this study. All times are estimates for 1x 96-well plate. Specific timings will vary with context. B. Schematic to illustrate the picking and REIMS scanning protocol following SCRaMbLE. C. 66 control (un-SCRaMbLEd, 'unSCR') strains and 405 SCRaMbLEd ('SCR') strains (top) were scanned by REIMS. BA ion count was normalised against an internal lipid peak $(835.55 \mathrm{~m} / \mathrm{z})$ for consistency when 
screening such large numbers of strains. Results expressed relative to the control mean. The top 20 strains (red) and bottom 19 (pink) strains were isolated and subjected to LCMS analysis in triplicate (bottom). Mean concentrations were established using a linear standard curve and normalised with $\mathrm{OD}_{600}$. Results are expressed relative to un-SCRaMbLEd control strains (dotted line, data not shown) that were run at the same time. Error bars indicate standard deviation.

\section{REIMS can detect a wide variety of biologically-relevant metabolites}

In this study LA-REIMS was used to screen for violacein and betulinic acid in negative ion detection mode. To investigate what other molecules LA-REIMS could screen for we prepared a series of standards covering a random selection of biological molecules relevant to synthetic biology researchers (Supplementary Table 5). Standards were spotted in duplicate onto yeast biomass in the same manner as shown in Fig 2A. Undiluted and a 1:10 dilution of standards were spotted and screened with LA-REIMS in both positive and negative ion detection modes with no optimisation for each metabolite. A positive result was recorded if the expected $\mathrm{m} / \mathrm{z}$ peak was seen and the ratio of the 1:10 dilution/undiluted was $<1$ to show that the peak of interest declined in relation to concentration. We screened a panel of 27 diverse metabolites, 4 were visible as negative ions only, 7 as positive ions only, 9 as both negative and positive ions, and 7 were not visible in either (Fig 4, Supplementary Table 5). Interestingly, the majority of the molecules undetectable by LA-REIMS lack an acid or basic group which would inherently challenge detection by any MS method without significant optimisation. These results indicate that LA-REIMS has the potential to semiquantitatively screen a number of biologically-relevant molecules diverse in molecular weight, hydrophobicity, charge, and metabolite class. 


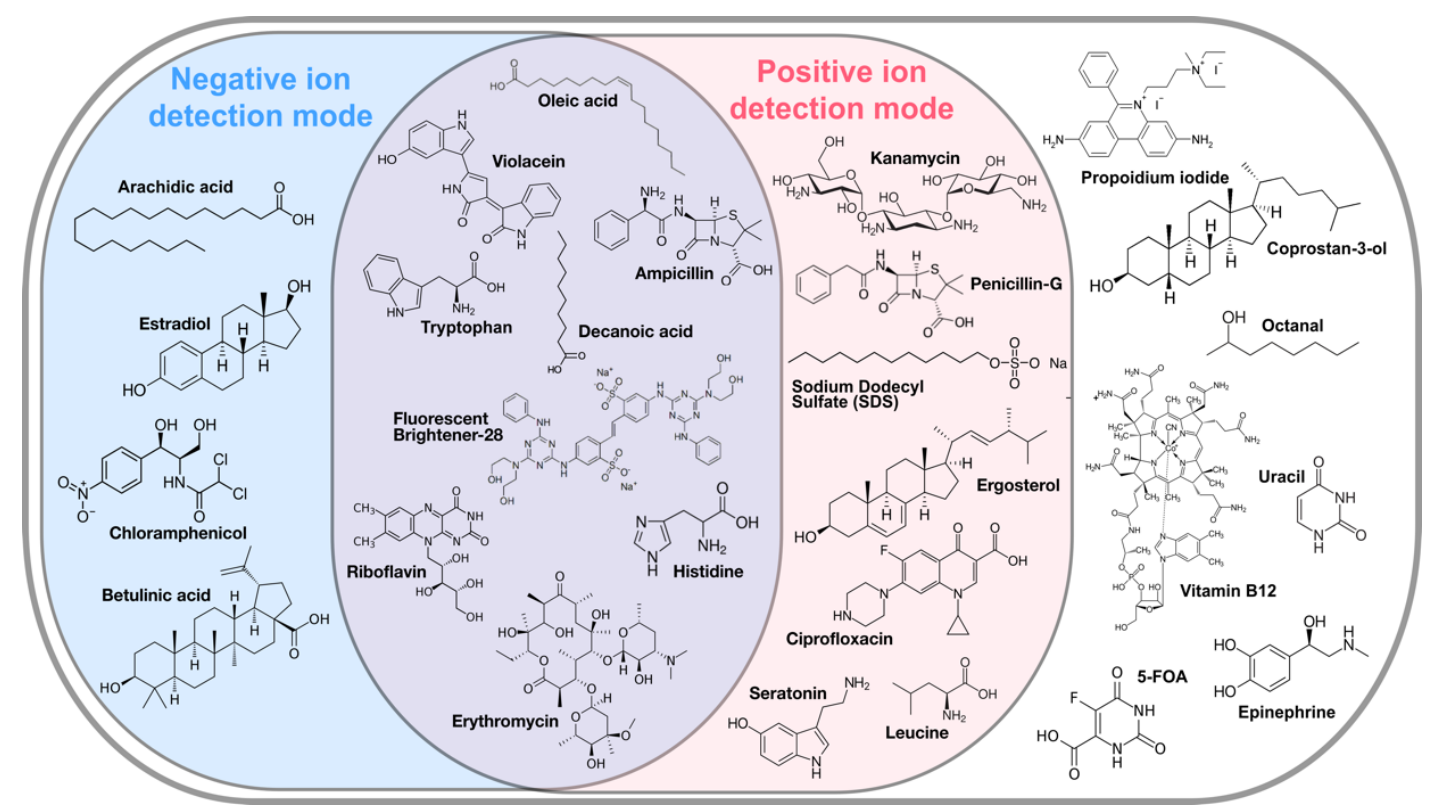

Fig.4 A selection of 27 metabolites screened by REIMS. A positive result in negative ion detection mode (blue) and positive ion detection mode (pink) are indicated with the overlapping area indicating a positive result in both ionisation modes. Metabolites in the white area indicate a negative result (see Supplementary Table 2)

\section{Discussion}

The development of rapid and simple-to-execute screening tools that offer higher throughput and fewer preparation steps are critical to relieving the major bottleneck in strain development. As demonstrated here, LA-REIMS offers a rapid and simple first-pass screen that can analyse $\sim 6$ colonies per minute (if picking is not required) and demands no sample preparation steps. As we have established here, throughput of approximately 1000 colonies a day is readily achievable with a picking and stocking step included. Using this approach, we showed that during a SCRaMbLE experiment LA-REIMS can screen hundreds of colonies and the top hits can then be subjected to LCMS analysis in triplicate. LA-REIMS, therefore, complements current quantitative screening tools. Importantly, despite a relatively high false negative rate where some high producers may be missed by LA-REIMS this workflow took significantly less time and effort than would have been required if we had performed LCMS analysis on all $400+$ strains. 
In its current incarnation, LA-REIMS satisfies requirements for a first-pass screening tool but cannot yet replace the gold standard of traditional LCMS or GCMS methods. The technique requires complex data pre-processing due to the lack of chromatographic separation and baseline noise. Furthermore, the limits of detection for various metabolites varies in a broad range using standard parameters. This is most acutely observed if the metabolite of interest has a mass in the $\mathrm{m} / \mathrm{z}$ range of endogenous lipids ( $600-900 \mathrm{~m} / \mathrm{z}$, Supplementary Figures $3 \mathrm{~A}$ and $4 \mathrm{~A}$ ). Fortunately, the confidence in semi-quantitative detection using LA-REIMS is greater at higher levels of the metabolite as the $\mathrm{m} / \mathrm{z}$ peak moves away from the noise of ambient ionisation mass spectrometry analysis. This property is synergistic with the demands of industrial strain engineering where higher levels of production are continuously sought, and low-level producers discarded. Additionally, in its current form LA-REIMS lacks a separation technique (such as ion mobility or MS/MS). While future work will aim to implement such techniques into LA-REIMS the lack of separation means species identification is limited to reference to a standard by $\mathrm{m} / \mathrm{z}$ only (Supplementary Table 6). As demonstrated in previous REIMS studies multiple metabolite peaks can be analysed simultaneously as entire spectra are obtained with no bias introduced by sample preparation ${ }^{41}$. Although not directly utilised in this study this capability would allow precursor and products of competing pathways to be analysed in real-time with the product of interest or in retrospect.

An interesting attribute of LA-REIMS, as presented here, is that it samples cells that are grown on solid-phase in colony form, whereas other traditional MS methods chiefly assess cells growing in liquid culture conditions. Previous studies analysis solid biomass for metabolite levels include DESI-MS ${ }^{23-28}$, LESA-MS ${ }^{29-31}$, MALDI-MS $^{32-34}$, and DI-MS ${ }^{35-37}$ but are limited in throughput, infrastructure requirements, sample preparation requirements or fundamental limitations such as only sampling extracellular metabolites (summarised in Supplementary Table 6). A universal drawback of sampling solid biomass, however, is that differences in the metabolic state of a yeast cell in a colony versus those growing in liquid 
are likely to play a role in seeing different production levels between strains sampled by methods such as LA-REIMS and by LCMS, especially for cells engineered to produce secondary metabolites like betulinic acid. We suspect that the majority of these differences stem from disparities in aeration, nutrient supply and growth phase. In shaking liquid culture cells are continuously and evenly aerated and are in constant contact with an even supply of nutrients leading to a largely homogenous culture. However, in colony form a non-uniform distribution of oxygen is assumed with the centre of the colony presumably being less aerated than the edges. Additionally, nutrients must diffuse up from the agar plane resulting in a non-uniform distribution of nutrients through the colony. We demonstrate here that translation of high-performing colonies (identified by LA-REIMS) to high-performing liquid culture strains (confirmed by LCMS) is subject to expected variation resulting in LA-REIMS giving both false positive and false negative outcomes, yet a handful of strains are still high producers in both conditions. It is worth noting, however, that downstream industrial applications will likely involve bioreactors within which cells grow at much higher densities than in lab-scale experiments ${ }^{58}$. At industrial scale growth, key conditions like oxygen availability and cell density will be very different from those seen in the aerated liquid growth used to prepare for standard lab LCMS assays. It may well be that colony-based growth, as assessed by LA-REIMS, adds a new dimension for assessment of strains suitable for scaleup. Furthermore, new emerging application of synthetic biology, such as engineering microbiomes and biofilms, will require identifying strains performing well when cell densities are very high ${ }^{59}$ and again, these may be closer to the conditions seen in colony growth.

We show here that LA-REIMS is a particularly useful tool to identify strains that are high producers in dissimilar environments. Future work will aim to characterise the performance of heterologous biosynthetic pathways under conditions of colony growth on agar versus shaking liquid growth as well as under commercial scale-up conditions. Furthermore, we show that LA-REIMS has the potential to screen for a number of biologically-relevant metabolites that may be targeted for industrial biosynthesis. The potential of screening by 
LA-REIMS is not just limited to yeast. Indeed, previous uses of REIMS indicate there are no inherent constraints on the type of biomass that can be screened, opening the door to extremely promising and diverse potential applications for screening by LA-REIMS.

\section{Acknowledgements}

We thank Leslie Mitchell and Jef Boeke for distribution of plasmid pJC104 and John Dueber for distribution of the MoClo YTK kit. We thank Dr Will Shaw for construction of preassembled YTK vectors. We also thank Dr Ben Blount, Marcus (Kenny) Walker, Dr Charlie Gilbert, Dr Will Shaw, and Dr Kealan Exley for useful discussions.

\section{Funding Sources}

This work was funded in the UK by BBSRC grants BB/P504579/1 and BB/L020858/1 and partly funded and supported by the Waters Corporation through provision of equipment and consumables and research funding to Imperial College London. GSK support GO.F.G through an industrial CASE studentship.

\section{Author information}

\section{Contributions}

G-O.F.G, S.J.S.C, and T.E conceived and designed the experiments. G-O.F.G and S.J.S.C performed the experiments and subsequent data analysis. Computational data analysis was assisted by A.P-M. G-O.F.G, D.B, and S.M.C designed and performed sample preparation for, and execution of, LCMS analysis. M.K, D.T, T.E, and Z.T assisted in interpreting the results. G-O.F.G, S.J.S.C, and T.E prepared and reviewed the manuscript.

\section{Conflicts of interest}

This work was partly funded ad supported by the Waters Corporation (through funding of Waters Corporation staff salaries, provision of equipment and consumables, and research funding to Imperial College London). Z.T. provides paid consultancy to the Waters 
Corporation. GSK support G-O.F.G through an industrial CASE studentship. No funders had input into initial study design nor on decision of when and where to publish this manuscript. This paper does not promote any commercially available product of the Waters Corporation, Ambimass or GSK. Z.T, A.P-M, and S.C are directors and persons of significant control at Ambimass.

\section{Supporting Information}

The Supporting Information is available free of charge on the ACS Publications website Supplementary Figure 1: Biosynthesis of violacein

Supplementary Figure 2: Biosynthesis of betulinic acid

Supplementary Figure 3: Mass spectra from REIMS (violacein)

Supplementary Figure 4: Mass spectra from REIMS (betulinic acid)

Supplementary Figure 5: Enrichment analysis for REIMS screen of violacein library

Supplementary Figure 6: Comparison of BA peak area only vs total ion count normalisation

Supplementary Figure 7: Location of betulinic acid extract

Supplementary Figure 8: LC-MS chromatogram for betulinic acid

Supplementary Table 1: List and sequence of genes used in this study for the biosynthesis of betulinic acid and violacein

Supplementary Table 2: List of plasmid cassettes used for assembly of biosynthetic pathways for violacein and betulinic acid

Supplementary Table 3: List of final plasmids that were transformed into yeast strains

Supplementary Table 4: List of yeast strains

Supplementary Table 5: List of metabolites screened using LA-REIMS

Supplementary Table 6: A comparison table of mass spectrometry technologies relevent to LA-REIMS 


\section{Online Methods}

\section{Strains and media}

S. cerevisiae strain BY4741 containing a synthetic chromosome V (yXZX846, 'synV') was created in a previous study ${ }^{53}$. For betulinic acid production CEN/ARS plasmids were transformed into yGG037 (synV with AtATR1 integrated into the HO locus with HIS+ auxotrophic marker). Plasmids used to create yGG037, the BA plasmid library, and the violacein library are listed in detail in Supplementary Tables 1-3). A full list of yeast strains are described in Supplementary Table 4. NEB ${ }^{\circledR}$ Turbo Competent Escherichia coli (NEB, MA, US) was used for bacterial cloning and plasmid propagation. Synthetic glucose media (SDO; $6.7 \mathrm{gL}^{-1}$ yeast nitrogen base, $1.4 \mathrm{gL}^{-1}$ yeast synthetic drop out medium supplement without histidine, leucine, tryptophan, and uracil, $20 \mathrm{gL}^{-1}$ glucose, $20 \mathrm{gL}^{-1}$ bacteriological agar (where appropriate)) was used for yeast growth supplemented with uracil (20 mgL-1), tryptophan $\left(20 \mathrm{mgL}^{-1}\right)$, leucine $\left(120 \mathrm{mgL}^{-1}\right)$ and histidine $\left(20 \mathrm{mgL}^{-1}\right)$ (Sigma Aldrich, $\left.\mathrm{MO}, \mathrm{US}\right)$ as appropriate.

\section{Plasmid construction and yeast transformation}

Plasmid for violacein and betulinic acid biosynthesis were constructed using the MoCloYeast Toolkit (YTK) ${ }^{56}$. Enzymes were supplied by NEB. Genes AtATR1, BPLO, AtLUS1, tHMG1 and ERG9 were codon optimised and synthesised by Thermo GeneArt (ThermoFisher, MA, US) with appropriate YTK overhang sequences (Supplementary Table 1). Genes vioA, vioB, vioC, vioD, and vioE were amplified from pJC104 to contain the appropriate YTK overhang sequences ${ }^{57}$ (Supplementary Table 1). AtATR1 was integrated into the HO locus of a synV strain to create yGG037 using linearised plasmid pGG127 ${ }^{48}$. Hierarchical construction of the plasmid libraries is indicated in Supplementary Table 2 and 3). The violacein plasmid library (plasmids pGG094 - pGG125) was transformed into synV to generate strains yGG074-yGG105 (Supplementary Figure 1 and Supplementary Table 4). The betulinic acid plasmid library (plasmids pGG024 - pGG059) was transformed into yGG037 to create strains yGG038-yGG073 (Supplementary Figure 2 and Supplementary 
Table 4). pSCW11-creEBD LEU+ was transformed into synV for SCRaMbLE induction.

Yeast transformations were performed using a standard lithium acetate protocol including a 17 minute $42^{\circ} \mathrm{C}$ heat shock step. Plasmids were isolated from $2 \mathrm{ml}$ saturated $E$. coli culture using QIAprep spin Miniprep Kits (Qiagen, Germany).

\section{SCRaMbLE}

Single colonies of yGG066 (BA) were picked and used to inoculate $5 \mathrm{ml} \mathrm{URA}^{-}$SDO media and grown overnight $\left(30^{\circ} \mathrm{C}, 250 \mathrm{rpm}\right)$. Each culture was used to inoculate two tubes of fresh $5 \mathrm{ml}$ selective media to $\mathrm{OD}_{600} \sim 0.2$ and grown for approximately $4 \mathrm{~h}$ to $\mathrm{OD}_{600} \sim 0.4-0.5$. At this time $1 \mu \mathrm{l} 5 \mathrm{mM} \beta$-estradiol (Sigma Aldrich, Merck, Darmstadt, Germany) was added to one tube (final concentration of $1 \mu \mathrm{M}$ ) with the second tube acting as an uninduced (unSCRaMbLEd) control. After induction cultures were grown for a further $4 \mathrm{~h}$ before being washed in deionised nuclease-free $\mathrm{H}_{2} \mathrm{O} .100 \mu \mathrm{l}$ of a $10^{-5}$ serial dilution (SCRaMbLEd) or $10^{-4}$ serial dilution (unSCRaMbLEd control) was plated on URA' selective agar and incubated at $30^{\circ} \mathrm{C}$ for $48 \mathrm{~h}$ prior to LA-REIMS analysis.

\section{LA-REIMS analysis}

Strains were either streaked directly onto selective media or picked into fresh selective media for $24 \mathrm{~h}$ shaking growth at $30^{\circ} \mathrm{C}$ before spotting $10 \mu \mathrm{l}$ onto agar media, as indicated in the text. Plates were grown for $48 \mathrm{~h}$ at $30^{\circ} \mathrm{C}$ prior to LA-REIMS analysis. Post-SCRaMbLE strains were analysed by LA-REIMS directly from the original plate without re-streaking. High-throughput LA-REIMS analysis was performed using a modified liquid handling robot (Freedom EVO platform, TECAN, Switzerland) combined with a plate imaging and colony picker platform (SciRobotics, Israel). Colony picking was required for LA-REIMS SCRaMbLE screening and was done using bespoke stainless-steel probe tips modified from a $200 \mu \mathrm{l}$ TECAN pure tip. Three racks of tips were rotated between picking, ethanol bath, drying to minimize consumable usage, and therefore running cost. During LA-REIMS analysis a helium-cooled FELS-25A CO 2 laser (OmniGuide, USA) operated at 2W in repeat 
SuperPulse pulsatile mode with a $40 \mathrm{~ms}$ on/off pulse cycle was used to rapidly heat and evaporate the biomass with the vapour aspirated towards the mass spectrometer under the instrument's native vacuum. Prior to entry into the ion guide of the mass spectrometer, the aspirate was mixed with 2-propanol containing leucine-enkephalin at a concentration of 0.1 $\mathrm{ng} / \mu \mathrm{L}$ at a flow rate of $200 \mu \mathrm{L} / \mathrm{min}$. This combination of solvent matrix and aspirated vapour then collided with a heated kanthal ribbon collision surface heated to $\approx 700^{\circ} \mathrm{C}$ to break apart ion clusters. Mass spectrometry (MS) analysis of the LA-REIMS vapor was completed using a Xevo G2-XS Q-ToF (Waters Corporation, Wilmslow, UK) in both negative and positive ion detection modes at 1 second per scan. The instrument underwent daily calibration of the time-of-flight mass analyser using sodium formate according to the manufacturer's standard protocol. BA was detected at m/z $455.35[\mathrm{M}-\mathrm{H}]-$ and violacein at $\mathrm{m} / \mathrm{z} 342.085[\mathrm{M}-\mathrm{H}]-$.

Unless otherwise stated, raw mass spectral data was subjected to lock mass correction and scan centroid detection for a total peak intensity calculation using sum utilising an in-house R script and Offline Model Builder software (Waters, Version 1.1.997.0). In the SCRaMbLE experiment, the intensity of a complex lipid peak (m/z 835.55 [M-H]-) was used as an internal standard to determine success of sample analysis when screening larger numbers of samples.

\section{Betulinic acid purification for LCMS analysis}

For the BA library biomass was picked into $100 \mu$ deionised water and centrifuged (3220 rcf 5 minutes) to obtain a pellet. For the SCRaMbLE strain analysis by LCMS the selected strains were streaked out from $-80^{\circ} \mathrm{C}$ stocks (picked during LA-REIMS analysis) onto selective URA ${ }^{-}$agar plates. Colonies were subsequently picked into $500 \mu \mathrm{U} \mathrm{URA}^{-}$selective media in a $2 \mathrm{ml}$ 96-deep well plate (Grenier Bio-One, Monroe, NC) and culture overnight at $30{ }^{\circ} \mathrm{C}, 700 \mathrm{rpm}$ with a Breathe-Easy ${ }^{\circledR}$ membrane (Sigma Aldrich). $5 \mu$ of this culture was used to inoculate $500 \mu$ fresh $\mathrm{URA}^{-}$selective media and incubated for a further $24 \mathrm{~h}\left(30{ }^{\circ} \mathrm{C}\right.$, $700 \mathrm{rpm}$ ). Each plate was centrifuged (3220 rcf, 5 minutes) to obtain a pellet. $200 \mu \mathrm{l}$ propan2-ol was added to each sample and vortexed for 2 minutes. $150 \mu$ l of supernatant was 
transferred to an LCMS 96-well plate (Agilent, CA, US) following centrifugation (3220 rcf, 10 minutes, $4^{\circ} \mathrm{C}$ ). A further $200 \mu \mathrm{l}$ propan-2-ol was added to the samples and similarly vortexed and centrifuged before $200 \mu \mathrm{l}$ supernatant was transferred to the same LCMS plate to yield a total volume of $350 \mu \mathrm{l}$ per sample. Each LCMS plate was dried down under nitrogen gas and resuspended in $70 \mu \mathrm{l}$ 2-propanol (VWR) to achieve a 1:5 concentrating effect.

\section{Betulinic acid quantification by LCMS}

Betulinic acid was detected and measured using an Agilent 1290 LC and 6550 Q-ToF mass spectrometer with electrospray ionization in negative polarity. The MS data acquisition rate was 10 spectra/second. The analysis was performed using Zorbax Eclipse Plus C18 UHPLC column (2.1 x 50 mm, $1.8 \mu \mathrm{m}$ particle size; Agilent Technologies, Santa Clara, CA, USA). LC buffers used were as follows: solvent $A, 50 \%$ of methanol in $0.1 \%(v / v)$ formic acid in water; solvent $\mathrm{B}, 0.1 \%(\mathrm{v} / \mathrm{v})$ formic acid in acetonitrile. Samples and standards were analysed at $0.2 \mu \mathrm{l}$ injection volume. The LC gradient is 0.5 minute in total with 1 minute post time run as summarized in below table. Quantitation by LC/MS was based on the peak area of accurately measured deprotonated betulinic acid, $[\mathrm{M}-\mathrm{H}]^{-}$, extracted with a window of $+/-$ $50 \mathrm{ppm}$.

\begin{tabular}{|c|c|c|}
\hline Time, $\min$ & Solvent $\mathrm{A}, \%$ & Solvent $\mathrm{B}, \%$ \\
\hline 0 & 98 & 2 \\
\hline 0.05 & 98 & 2 \\
\hline 0.80 & 2 & 98 \\
\hline 0.90 & 2 & 98 \\
\hline 0.91 & 98 & 2 \\
\hline 1.20 & 98 & 2 \\
\hline
\end{tabular}




\section{References}

1. Ro, D. et al. Production of the antimalarial drug precursor artemisinic acid in engineered yeast. Nature 440, 3-6 (2006).

2. Galanie, S., Thodey, K., Trenchard, I. J., Interrante, M. F. and Smolke, C. D. Complete biosynthesis of opioids in yeast. Science 349, 1095-1100. (2015).

3. Awan, A. R., Blount, B. A., Bell, D. J., Shaw, W. M., Ho, J. C. H., McKiernan, R. M., and Ellis, T. Biosynthesis of the antibiotic nonribosomal peptide penicillin in baker's yeast. Nat. Commun. 8. (2017)

4. Billingsley, J. M., DeNicola, A. B. and Tang, Y. Technology development for natural product biosynthesis in Saccharomyces cerevisiae. Curr. Opin. Biotechnol. 42, (2016).

5. Marienhagen, J. and Bott, M. Metabolic engineering of microorganisms for the synthesis of plant natural products. J. Biotechnol. 163, 166-178 (2013).

6. Meadows, A. L. et al. Rewriting yeast central carbon metabolism for industrial isoprenoid production. Nature 537, 694-697 (2016).

7. Hughes, R. A. and Ellington, A. D. Synthetic DNA synthesis and assembly: Putting the synthetic in synthetic biology. Cold Spring Harbor Perspect. Biol. (2017).

8. Bao, Z. et al. Genome-scale engineering of Saccharomyces cerevisiae with singlenucleotide precision. Nat. Biotechnol. 36, (2018).

9. Shepelin, D., Hansen, A., Lennen, R., Luo, H. and Herrgård, M. Selecting the Best: Evolutionary Engineering of Chemical Production in Microbes. Genes 9, 249 (2018).

10. Dymond, J. S. et al. Synthetic chromosome arms function in yeast and generate phenotypic diversity by design. Nature 477, 471-6 (2011).

11. Blount, B. A. et al. Rapid host strain improvement by in vivo rearrangement of a synthetic yeast chromosome. Nat. Commun. (2018).

12. Luo, Z. et al. Identifying and characterizing SCRaMbLEd synthetic yeast using ReSCuES. Nat. Commun. 9, 1-10 (2018).

13. Wang, J. et al. Ring synthetic chromosome V SCRaMbLE. Nat. Commun. 9, 3783 
(2018).

14. Wu, Y. et al. In vitro DNA SCRaMbLE. Nat. Commun. 9, (2018).

15. Hochrein, L., Mitchell, L. A., Schulz, K., Messerschmidt, K. and Mueller-Roeber, B. LSCRaMbLE as a tool for light-controlled Cre-mediated recombination in yeast. Nat. Commun. 9, 1-10 (2018).

16. Jia, B. et al. Precise control of SCRaMbLE in synthetic haploid and diploid yeast. Nat. Commun. 9, (2018).

17. Shen, M. J. et al. Heterozygous diploid and interspecies SCRaMbLEing. Nat. Commun. 9, (2018).

18. Liu, W. et al. Rapid pathway prototyping and engineering using in vitro and in vivo synthetic genome SCRaMbLE-in methods. Nat. Commun. 9, 1-12 (2018).

19. Petzold, C. J., Chan, L. J. G., Nhan, M. and Adams, P. D. Analytics for Metabolic Engineering. Front. Bioeng. Biotechnol. 3, 1-11 (2015).

20. Liu, D., Evans, T. and Zhang, F. Applications and advances of metabolite biosensors for metabolic engineering. Metab. Eng. 31, 15-22 (2015).

21. Carpenter, A. C., Paulsen, I. T. and Williams, T. C. Blueprints for biosensors: Design, limitations, and applications. Genes 9, 375 (2018).

22. Terekhov, S. S. et al. Microfluidic droplet platform for ultrahigh-throughput single-cell screening of biodiversity. Proc. Natl. Acad. Sci. U. S. A. (2017).

23. Yan, C. et al. Real-time screening of biocatalysts in live bacterial colonies. J. Am. Chem. Soc. 139, 1408-1411 (2017).

24. Song, Y., Talaty, N., Tao, W. A., Pan, Z. and Cooks, R. G. Rapid ambient mass spectrometric profiling of intact, untreated bacteria using desorption electrospray ionization. Chem. Commun. (Cambridge, U. K.) 61-63 (2007).

25. Esquenazi, E., Dorrestein, P. C. and Gerwick, W. H. Probing marine natural product defenses with DESI-imaging mass spectrometry. Proc. Natl. Acad. Sci. U. S. A. 106, 7269-7270 (2009).

26. Roach, P. J., Laskin, J. and Laskin, A. Nanospray desorption electrospray ionization: 
An ambient method for liquid-extraction surface sampling in mass spectrometry. Analyst (Cambridge, U. K.) 135, 2233-2236 (2010).

27. Lanekoff, I., Geydebrekht, O., Pinchuk, G. E., Konopka, A. E. and Laskin, J. Spatially resolved analysis of glycolipids and metabolites in living Synechococcus sp. PCC 7002 using nanospray desorption electrospray ionization. Analyst (Cambridge, U. K.) 138, 1971-1978 (2013).

28. Hartmanova, L. et al. Fast profiling of anthocyanins in wine by desorption nanoelectrospray ionization mass spectrometry. J. Chromatogr. A 1217, 4223-4228 (2010).

29. Kocurek, K. I., Stones, L., Bunch, J., May, R. C. and Cooper, H. J. Top-Down LESA Mass Spectrometry Protein Analysis of Gram-Positive and Gram-Negative Bacteria. J. Am. Soc. Mass Spectrom. 28, 2066-2077 (2017).

30. Eikel, D. et al. Liquid extraction surface analysis mass spectrometry (LESA-MS) as a novel profiling tool for drug distribution and metabolism analysis: The terfenadine example. Rapid Commun. Mass Spectrom. 25, 3587-3596 (2011).

31. Sarsby, J. et al. Liquid Extraction Surface Analysis Mass Spectrometry Coupled with Field Asymmetric Waveform Ion Mobility Spectrometry for Analysis of Intact Proteins from Biological Substrates. Anal. Chem. 87, 6794-6800 (2015).

32. Si, T. et al. Profiling of Microbial Colonies for High-Throughput Engineering of Multistep Enzymatic Reactions via Optically Guided Matrix-Assisted Laser Desorption/lonization Mass Spectrometry. J. Am. Chem. Soc. 139, 12466-12473 (2017).

33. Devereaux, Z. J. et al. Matrix-assisted ionization on a portable mass spectrometer: Analysis directly from biological and synthetic materials. Anal. Chem. 88, 1083110836 (2016).

34. Bian, J. and Olesik, S. V. Surface-assisted laser desorption/ionization time-of-flight mass spectrometry of small drug molecules and high molecular weight synthetic/biological polymers using electrospun composite nanofibers. Analyst 
(Cambridge, U. K.) 142, 1125-1132 (2017).

35. Lim, D. K., Mo, C., Long, N. P., Kim, G. and Kwon, S. W. Simultaneous profiling of lysoglycerophospholipids in rice (Oryza sativa L) using direct infusion-tandem mass spectrometry with multiple reaction monitoring. J. Agric. Food Chem. 65, 2628-2634 (2017).

36. Castrillo, J. I., Hayes, A., Mohammed, S., Gaskell, S. J. and Oliver, S. G. An optimized protocol for metabolome analysis in yeast using direct infusion electrospray mass spectrometry. Phytochemistry 62, 929-937 (2003).

37. Ernst, L., Langer, N., Bockelmann, A., Salkhordeh, E. and Beuerle, T. Identification and quantification of synthetic cannabinoids in 'spice-like' herbal mixtures: Update of the German situation in summer 2018. Forensic Sci. Int. 294, 96-102 (2019).

38. Takats, Z., Strittmatter, N. and McKenzie, J. S. Ambient Mass Spectrometry in Cancer Research. Adv. Cancer Res. 134 (2017).

39. Balog, J. et al. Identification of biological tissues by rapid evaporative ionization mass spectrometry. 82, 7343-7350 Anal. Chem. (2010).

40. Bolt, F. et al. Automated High-Throughput Identification and Characterization of Clinically Important Bacteria and Fungi using Rapid Evaporative Ionization Mass Spectrometry. 88, 9419-9426 Anal. Chem. (2016).

41. Cameron, S. J. S. et al. Rapid Evaporative Ionisation Mass Spectrometry (REIMS)

Provides Accurate Direct from Culture Species Identification within the Genus Candida. Sci. Rep. 6, 36788 (2016).

42. Bardin, E. E. et al. Metabolic Phenotyping and Strain Characterisation of Pseudomonas aeruginosa Isolates from Cystic Fibrosis Patients Using Rapid Evaporative Ionisation Mass Spectrometry. Sci. Rep. 8, 10952 (2018).

43. Cameron, S. J. S. et al. Utilisation of Ambient Laser Desorption Ionisation Mass Spectrometry (ALDI-MS) Improves Lipid-Based Microbial Species Level Identification. Sci. Rep. 9, 1-8 (2019).

44. Schäfer, K. C. et al. In situ, real-time identification of biological tissues by ultraviolet 
and infrared laser desorption ionization mass spectrometry. Anal. Chem. 83, 1632$1640(2011)$

45. Gali-Muhtasib, H., Hmadi, R., Kareh, M., Tohme, R. and Darwiche, N. Cell death mechanisms of plant-derived anticancer drugs: Beyond apoptosis. Apoptosis 20, $1531-1562(2015)$.

46. Aiken, C. and Chen, C. H. Betulinic acid derivatives as HIV-1 antivirals. Trends Mol. Med. 11, 31-36 (2005).

47. Saha, S., Ghosh, M. and Dutta, S. K. A potent tumoricidal co-drug 'Bet-CA'--an ester derivative of betulinic acid and dichloroacetate selectively and synergistically kills cancer cells. Sci. Rep. 5, 7762 (2015).

48. Zhou, C., Li, J., Li, C. and Zhang, Y. Improvement of betulinic acid biosynthesis in yeast employing multiple strategies. BMC Biotechnol. 16, 59 (2016).

49. Sun, Y., Sun, L., Shang, F. and Yan, G. Enhanced production of $\beta$-carotene in recombinant Saccharomyces cerevisiae by inverse metabolic engineering with supplementation of unsaturated fatty acids. Process Biochem. (Oxford, U. K.) 51, 568577 (2016)

50. Kennedy, M. A., Barbuch, R. and Bard, M. Transcriptional regulation of the squalene synthase gene (ERG9) in the yeast Saccharomyces cerevisiae. Biochim. Biophys. Acta, Gene Struct. Expression 1445, 110-122 (1999).

51. Thompson, A., Kwak, S. and Jin, Y.-S. Squalene Production using Saccharomyces cerevisiae. i-ACES 1, 57-63 (2014).

52. Xie, W., Lv, X., Ye, L., Zhou, P. and Yu, H. Construction of lycopene-overproducing Saccharomyces cerevisiae by combining directed evolution and metabolic engineering. Metab. Eng. 30, 69-78 (2015).

53. Xie, Z.-X. et al. 'Perfect' designer chromosome $\mathrm{V}$ and behavior of a ring derivative. Science 355 (2017).

54. Dymond, J. and Boeke, J. The Saccharomyces cerevisiae SCRaMbLE system and genome minimization. Bioengineered 3, 168-171 (2012). 
55. Richardson, S. M. et al. Design of a synthetic yeast genome. Science 355, 1040$1044(2017)$.

56. Lee, M. E., DeLoache, W. C., Cervantes, B. and Dueber, J. E. A Highly Characterized Yeast Toolkit for Modular, Multipart Assembly. ACS Synth. Biol. 4, 975-986 (2015).

57. Mitchell, L. A. et al. Versatile genetic assembly system (VEGAS) to assemble pathways for expression in S. cerevisiae. Nucleic Acids Res. 43, 6620-6630 (2015).

58. Westman, J. O., and Franzén, C. J. Current progress in high cell density yeast bioprocesses for bioethanol production. Biotechnol. J. 10, 1185-1195 (2015)

59. Flemming, H. C., et al. Biofilms: An emergent form of bacterial life. Nat. Rev. Microbiol. 14, 563-575 (2016) 
For Table of Contents Only

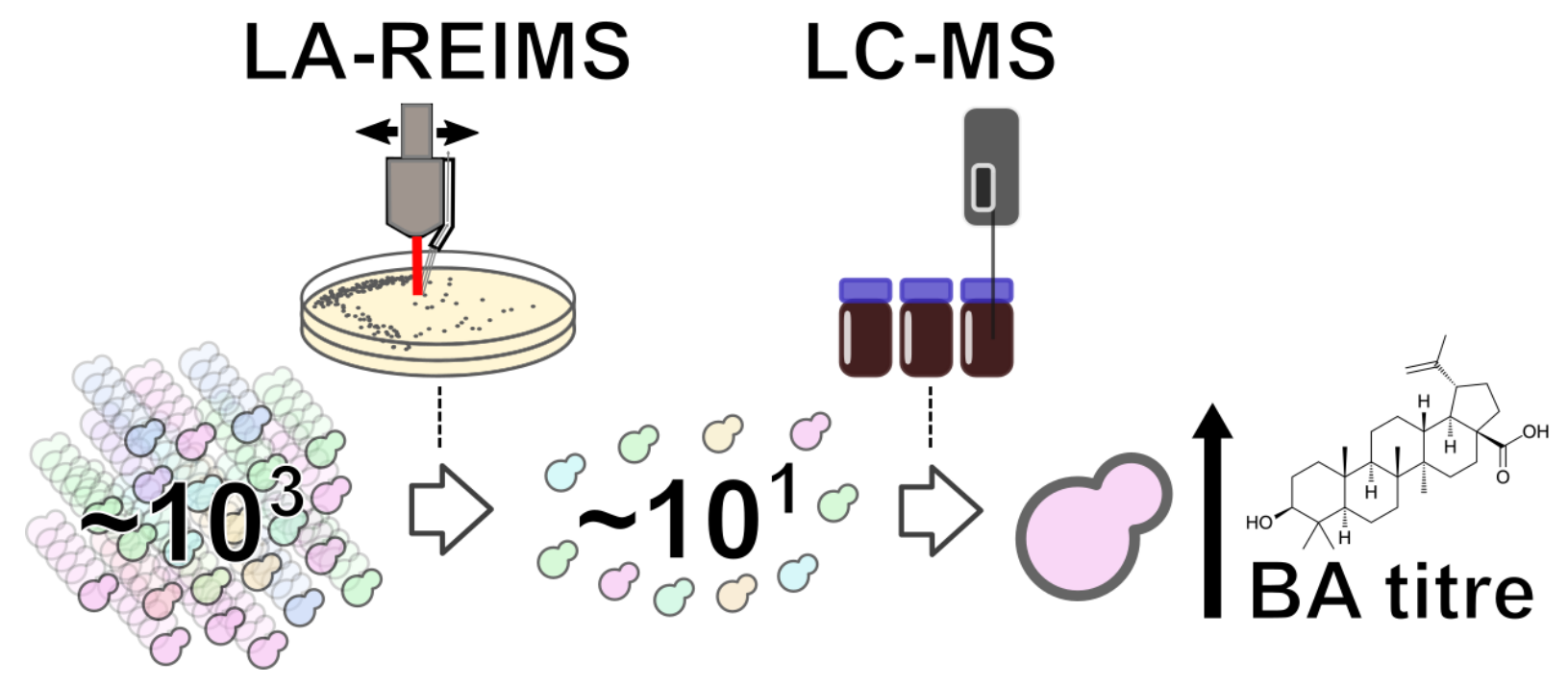

